\title{
Rain prediction using fuzzy rule based system in North-West Malaysia
}

\author{
Noor Zuraidin Mohd Safar ${ }^{1}$, Azizul Azhar Ramli ${ }^{2}$, Hirulnizam Mahdin ${ }^{3}$, David Ndzi ${ }^{4}$, \\ Ku Muhammad Naim Ku Khalif ${ }^{5}$ \\ 1,2,3 Faculty of Computer Science and Information Technology, Universiti Tun Hussein Onn Malaysia, Malaysia \\ ${ }^{4}$ School of Engineering and Computing, University of the West of Scotland, United Kingdom \\ ${ }^{5}$ Faculty of Industrial Sciences and Technology, Universiti Malaysia Pahang, Malaysia
}

\begin{tabular}{|c|c|}
\hline Article Info & ABSTRACT \\
\hline Article history: & \multirow{10}{*}{$\begin{array}{l}\text { The warm and humid condition is the characteristic of Malaysia tropical } \\
\text { climate. Prediction of rain occurrences is important for the daily operations } \\
\text { and decisions for the country that rely on agriculture needs. However } \\
\text { predicting rainfall is a complex problem because it is effected by the } \\
\text { dynamic nature of the tropical weather parameters of atmospheric pressure, } \\
\text { temperature, humidity, dew point and wind speed. Those parameters have } \\
\text { been used in this study. The rainfall prediction are compared and analyzed. } \\
\text { Fuzzy Logic and Fuzzy Inference System can deal with ambiguity that often } \\
\text { occurred in meteorological prediction; it can easily incorporate with expert } \\
\text { knowledge and empirical study into standard mathematical. This paper will } \\
\text { determine the dependability of Fuzzy Logic approach in rainfall prediction } \\
\text { within the given approximation of rainfall rate, exploring the use of Fuzzy } \\
\text { Logic and to develop the fuzzified model for rainfall prediction. } \\
\text { The accuracy of the proposed Fuzzy Inference System model yields } 72 \%\end{array}$} \\
\hline Received Dec 15, 2018 & \\
\hline Revised Feb 13, 2019 & \\
\hline Accepted Feb 27, 2019 & \\
\hline Keywords: & \\
\hline Expert system & \\
\hline Fuzzy & \\
\hline Fuzzy inference system & \\
\hline Meteorology & \\
\hline Rain prediction & \\
\hline Rainfall forecast & \\
\hline Rainfall prediction & $\begin{array}{r}\text { Of Aavancea Engineering ana sctence. } \\
\text { All rights reserved. }\end{array}$ \\
\hline Soft computing & \\
\hline Tropical climate & \\
\hline
\end{tabular}

Corresponding Author:

Noor Zuraidin Mohd Safar,

Faculty of Computer Science and Information Technology,

Universiti Tun Hussein Onn Malaysia,

86400 Parit Raja, Batu Pahat, Johor, Malaysia.

Email: zuraidin@uthm.edu.my

\section{INTRODUCTION}

Climate change has increased incidences of unusual rainfall pattern which often cause wide spread flooding. Any system that can provide advanced prediction of precipitation rates could be used to predict flooding before the rain event. The necessity for accurate local rainfall prediction is readily ostensible when considering many benefits of such information would provide for river control, reservoir operations, forestry interests, flash flood monitoring and agriculture needs. Data fusion provides new opportunities in rain and flood prediction. This is vital in tropical climates where rain is localized and flash flooding is common. Fusion of local environmental data such as temperature, humidity, atmospheric pressure, air pressure, dew point, solar radiation, cloud, wind direction and wind speed from sensors could potentially be used to provide more accurate local predictions.

The intention of this study is to recognize the rules and modeling rain prediction using Fuzzy Logic (FL) to predict rainfall. The rules will apply to forecast the rainfall in the Perlis (the northwest region of Malaysia). Basic environmental parameters including temperature, humidity, atmospheric pressure, dew point and wind speed are use in identifying the inference rules. FL is one of the core methodologies in soft computing which its purpose to exploit the tolerance for imprecise, uncertain, ambiguity and vagueness of the decision making. 
The implementation of soft computing in engineering and science can resolve problems that traditionally have not been able to be solved by analytical methods. The availability of required data to make such predictions has been available for quite some time. The complex and changes in relationships among the data and its effect on the probability has often proved difficult using conventional computer analysis. The use of soft computing technique is to learn rather than analyses these complex relationships, has shown a great deal of promise in accomplishing the goal of predicting both the probability and quantity of rain in a local area. The methodologies in soft computing include knowledge processing and flexible knowledge acquisition by learning from existing data to solve non-linear problem. Study shows that FL can achieved 70\% accuracy in data mining performance comparison [1]. The performance results are determined by assessing the magnitude of the error and the measurement of the correlation between the observed and forecasted values. Results showed that an hour ahead forecasts produce good outcomes. The outcomes indicate that Fuzzy Inference System models offer an ability to forecast a rainfall. This paper is structured as follows: Section 2 describes the literature and previous study, Section 3 describes the study area and the methodology. Section 4 presents the results and discussions and finally, Section 5 is the conclusion

\section{LITERATURE}

\subsection{Related Works}

Examples on the applications of soft computing based on FL in rainfall forecasting are numerous. Fuzzy Rule Based System (FBS) and Fuzzy Inference System (FIS) for example have been used experimentally to predict weather events such as rain, fog, tornado, thunderstorm or clear condition. Fuzzy Time Series (FTS) presented in the time invariant and variant model for dealing with forecasting problems was proposed in [1]. If the annual rainfall data spanning over more than ten years, the FTS model can be used as an appropriate forecasting tool to predict the rainfall [2]. The design and development of the rules based on the three main sources; expert opinion, literature survey and automated rule generation [3]. The rule base is commonly obtained from an expert opinion, Handoyo and Marji proposed the rule base generated based on input output pairs relationship and then each rule is optimized by ordinary least square (OLS) method [4]. Agboola et al. forecast the rainfall based on FIS technique. The system is entail of two useful modules; the knowledge base and the fuzzy reasoning unit. Two processes were executed on the FL model; the fuzzification and defuzzification operation [5]. Asklany et al. forecast rainfall with the help of FL based approximate reasoning, the IF-THEN rules. The fuzzy inference engine uses these fuzzy IF-THEN rules to determine a mapping from the input fuzzy sets to the output fuzzy sets based on fuzzy logic principles. The proposed model has the fuzzy sets contain of five environmental data: humidity, cloud, wind direction, temperature and air surface pressure. Those parameters are the input variables. Each fuzzy set has three membership functions with single output. In the fuzzy set theory, features of a fuzzy set are mapped to a universe of membership values using a function theoretic form belonging to the closed interval between 0 to 1 [6]. Hasan et al. developed the rainfall prediction model using selected fuzzy variables based on the degree of association of different factors. The influantial in wind speed and temperature were found to have a positive relation with rainfall but the proposed model showed enhanced performance after adding the threshold values of humidity [7]. Recently, Cristani et al. use fuzzy reasoning to formulaise an architecture that implements the reasoning techniques in environmental study [8]. The list of rainfall prediction models developed using FL approached is not exhaustive. Research and achievement of the model can be found in [9]-[15]. The above cases are just a few examples to illustrate the potential and future direction of rainfall forecasting model using FL.

\subsection{Fuzzy Logic and Fuzzy Inference System}

FL is a way to definite conclusion based upon vague, ambiguous, imprecise, noise or missing input information [16]. FL is good at reasoning with imprecise information and explaining their behaviour based on fuzzy rules but they cannot automatically acquire the rules they use for making decisions. It is attempts to mimic human thought process. Humans analyse situations and solve problems in a relatively imprecise manner, do not have all the facts and the facts might be uncertain. People in a norm, generalize facts without having the precise data or measurements. In such situation Fuzzy approach based on FL is seems to be suitable. In general FL offers an inference structure that enables suitable human reasoning competencies. The origin of FL approach was first introduced by Lotfi Zadeh in 1965 [16]. FL is valuable in modelling complex and imprecise systems. Fuzzy set theory is a powerful tool and its applications have rapidly increased with establishing its utility in numerous areas of the scientific exploration. Fuzzy set has degree of membership as opposed to traditional set theory where set of membership is defined as Boolean predicate. Fuzzy set theory defines set membership as a possibility distribution. The general rule for this can be expressed as: 


$$
f:[0,1]^{n} \rightarrow[0,1]
$$

Where $n$ is some number of possibilities and $n$ possible events and us $\mathrm{f}$ to generate as single possible outcome. The probability of FL outcome and its amount of result due to the vague input variables are being generated in the human mind and it is often stated as expert knowledge. This expert knowledge is the gathering of knowledge and ideas as a result of the experts' experience, judgement and individual knowledge. In a particular system, the processes of decision making may be considered as fuzzy expressions seemingly adopt by the experts [7].

Basic structure of Fuzzy Logic model with inreference engine is presented in Figure. It is consist of three main steps; Fuzzification Process, Fuzzy Rules and Inference Engine Determination and Defuzzification Process.

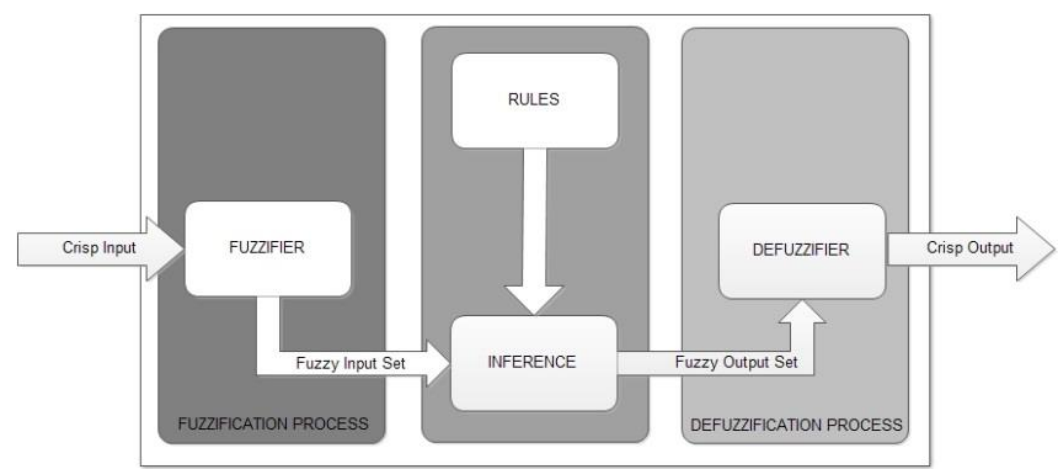

Figure 1. Basic structure of Fuzzy Logic model

\section{STUDY AREA}

The selected area is Perlis, one of the states in Malaysia. Perlis has a 13.33 kilometre square lake named Timah Tasoh. There are two main rivers which are Tasoh River and Pelarit River that flows into the Timah Tasoh lake [17]. Agriculture area in its upstream catchments and timber plantations are surrounding the lake. Infrastructures development of urbanizations involved land clearing activities and amount of rain influenced the river water discharge of the river. Timah Tasoh Lake can be classified as medium size and shallow lake [18]. Existing study of environmental forecasting within stipulated area using soft computing approach have been conducted in [19], [20]. Three years of environmental data is recorded in hourly basis. The sample space for constructing the rain predictions consisted of 4624 dataset from 2012 to 2014. $80 \%$ of the data is used for the training and the remaining $20 \%$ for testing and validation. The training data consists of 153 rainfall rate reading with the minimum value of $0.02 \mathrm{mh}^{-1}$ and the maximum value of $3.10 \mathrm{mh}^{-1}$. Map of Malaysia and chuping as shown in Figure 2.

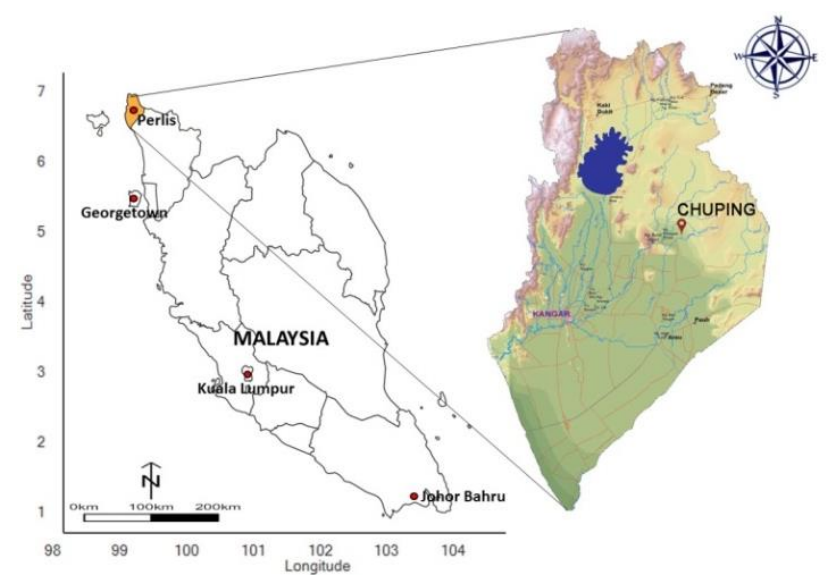

Figure 2. Map of Malaysia and chuping 


\section{APPLICATION OF RAINFALL FORECASTING}

\subsection{FIS Preliminary Implementation}

FIS is designed to work with the input parameters. Once input is given by the user, appropriate rule for the output is given by the system. The design process for the fuzzy model will make use of the fuzzy logic tool box in MATLAB [21]. Figure is the graphical illustration of the proposed fuzzy model includes five input parameters (Humidity, Temperature, Pressure, Wind Speed and Dew Point) and one output parameter (Rainfall Rate).

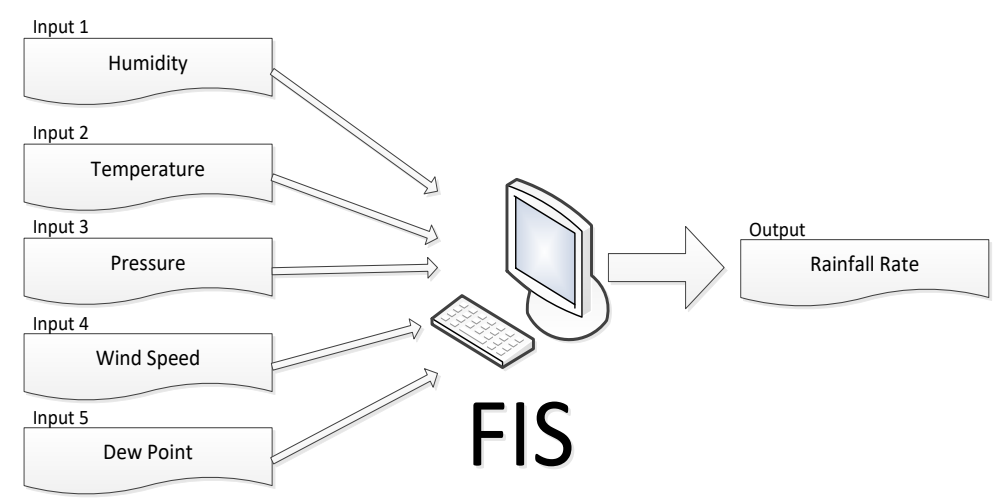

Figure 3. Schematic illustration of the rainfall rate fuzzy model

For each input and output variable selected, two or more membership functions (MF) is define, normally three but can be more qualitative category for each one of them. The proposed FIS will use four MFs while the output will use five MFS. The shape of these functions can be diverse but it is usually works with triangles. Once the input and output variables and MF are defined, the following step is to design the rule-base or decision matrix of the fuzzy knowledge-base that composed of expert IF THEN rules. These rules transform the input variables to an output that will tell the probability of rainfall rate. The output variables also have to be defined with MF, usually low, normal and high. Depending on the number of MF for the input and output variables the rule based will be able to define more or less potential of the existence of rain. The more variables existed, the more rules available to be defined, then it will make the inferences rules reliable. Once the realistic rules are defined according to the expert knowledge and based on collected weather data, these rules will become the knowledge base of each of the prediction model but it is not necessary to say that the whole knowledge to be translated in rules, sometimes some of the rules can be redundant or eliminated. This study uses the FIS Mamdani Model [22]. The Mamdani fuzzy model is similar to the Sugeno and Tsukomoto model where they have a set of inputs which each of the input has its own MFs. The Mamdani model differs from the others in the fact that the output also has a set of its own membership functions. In the case of the Sugeno model, the output is a function of the input MF and in the case of Tsukomoto's model; the output is a linear function of the input.

\subsection{Fuzzification}

Fuzzification is a process of transforming a real scalar value into a degree of membership (fuzzifier) in a particular fuzzy set value. The processes also do the mapping of the crisp input into fuzzy variable (linguistic label). Fuzzification of linguistic variables involves with the classification of parameters into linguistic labels and assigning MF for each of the variable.

\subsubsection{Determination of Membership Function}

The required data for the analysis of rainfall and construct the rules is collected from Chuping weather station. Three years of environmental data that consists of 4624 dataset from 2012 to 2014 have been used in this study. $80 \%$ of the data is used for the training and the remaining $20 \%$ for testing and validation. The training data consists of 153 rainfall rate reading with the minimum value of $0.02 \mathrm{mh}^{-1}$ and the maximum value of $3.10 \mathrm{mh}^{-1}$. From the schematic illustration in Error! Reference source not found., five input parameters (Humidity, Temperature, Pressure, Wind Speed and Dew Point) and one output parameter (Rainfall Rate) is used to develop the fuzzy model. In order to apply Fuzzy Logic, the parameters must be characterized properly by flexible membership function. 


\subsubsection{Elemntery Prediction Using IF-THEN Rules}

Elementary forms of prediction can be constructed using a policy based on simple IF THEN rules especially for strong inter-dependent conditions. The process of constructing the rules is to identify the combination of terms in the rule antecedent that best identify a class term. The combination rules can be implemented using the most common logical operator like AND and OR. The initial study was to identify the environmental parameters dependency in conjunction with rain condition.

Cumulative Distribution Function (CDF) curves were used to determine the most significant parameters to predict whether it is raining or not. The CDF of each parameter is compared between rain and no rain events. Figureshows the curves of humidity. When it was raining, humidity varied from $68 \%$ to $100 \%$. Thus the following rules is developed, IF Humidity $<70 \%$ THEN 'No Rain'. The rule constructed is IF Temperature $>33^{\circ}$ Celsius THEN 'No Rain' based on temperature's CDF.

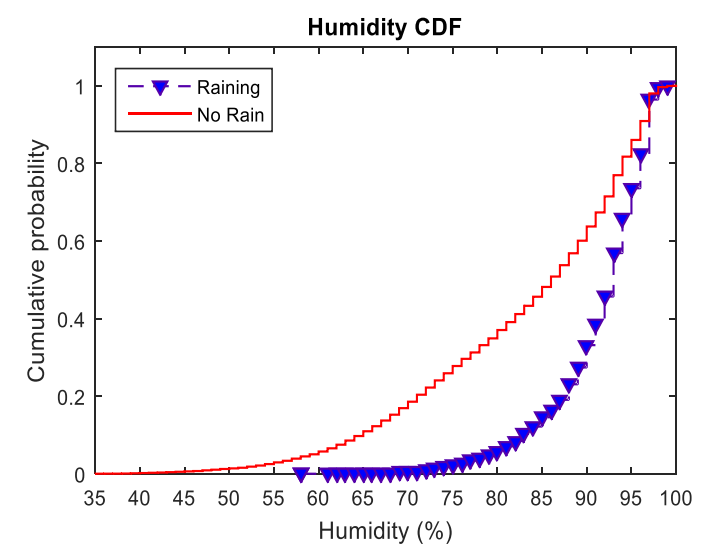

(a)

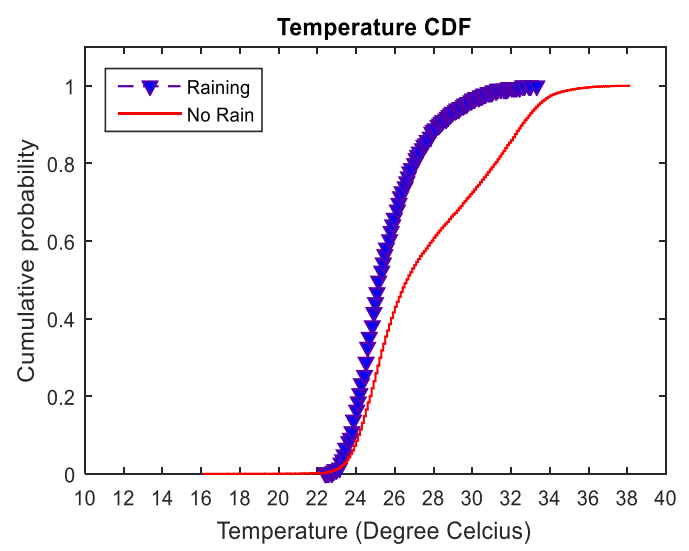

(b)

Figure 4. (a) CDF for Humidity, (b) CDF for Temperature

The following rules are constructed based on the analysis of CDF for each environmental parameter is given below:

\section{Rule A:}

IF

(Pressure $>=1014$ AND

Pressure < 1017) OR

(Humidity < 58) OR

(Temperature $<33$ ) OR

(Dew Point $>28)$

(Wind Speed $>=4$ AND

Wind Speed $<=10$ )

THEN (Prediction event is NO RAIN)
Rule B:

IF (Pressure < 1014) OR

(Humidity > 58) OR

(Temperature < 33 ) OR

(Dew Point $>21$ AND Dew Point $<28$ ) OR

(Wind Speed > 2.5) OR

THEN (Prediction event is RAIN)

The IF THEN rules prediction were constructed through analysing the training data. Rule $A$ is used to predict no-rain event and Rule $B$ to predict rain event. Rule $A$ and Rule $B$ are used to further develop fuzzy variables to feed the FIS prediction model. When rain event is detected, the ensuing question is about the rain intensity. In the following section the process of mapping between the fuzzy input and fuzzy output to predict rainfall rate is described.

\subsubsection{Input and Output Parameters}

The output parameter in Mamdani fuzzy model has a set of MF. In the proposed model a single output of the rainfall prediction with five MFs is expected after the appropriate fuzzification of inputs, inference rules and aggregation process. Details of the fuzzy model output will be discussed in the defuzzification process. The input parameters in this system are depicted in Table 1. The parameters is classified into linguistic description based on the piecewise MFs linguistic variable representation criteria [23]. 
Table 1. List of Input Parameters

\begin{tabular}{cccccc}
\hline Input Parameters & Unit & & Fuzzy Variables \\
\hline Humidity & $\%$ & Poor & Low & Medium & High \\
Temperature & ${ }^{o} \mathrm{C}$ & Poor & Low & Medium & High \\
Pressure & milibar & Poor & Low & Medium & High \\
Wind Speed & $\mathrm{Km} / \mathrm{h}$ & Poor & Low & Medium & High \\
Dew Point & ${ }^{\circ} \mathrm{C}$ & Poor & Low & Medium & High \\
\hline
\end{tabular}

A fuzzy set contains elements that have varying degrees of MF in the set of ordered pairs given by:

$$
\left.A=\left\{\left(x, \mu_{A}(x)\right) \mid \forall x \in X\right)\right\}
$$

Where $X$ a universal set and $\mu_{A}(x)$ (usually $0 \leq \mu_{A}(x) \leq 1$ ) is the grade of MF of the object $x$ in $A$. A membership function $\mu_{A}(x)$ is characterized by $\mu_{A}: X \rightarrow(0,1)$ where $X$ is the universe of discourse; $x$ is a real number describing an object or its attribute and each element of $X$ is mapped to a value between 0 and 1 [24]. MFs can be graphically represented a fuzzy set in the form of a triangular, trapezoidal or bell shape (Gaussian). Every input is fuzzified by constructing the MF. In the proposed model, a triangular membership functions were used to describe input and output variables. Triangular membership have been used because of their simplicity and computational efficiency [25]. The triangular MFs are shown in Figure where $a, b$ and $c$ are the parameters of the linguistic value and the $x$ axis is a value input parameter's series.

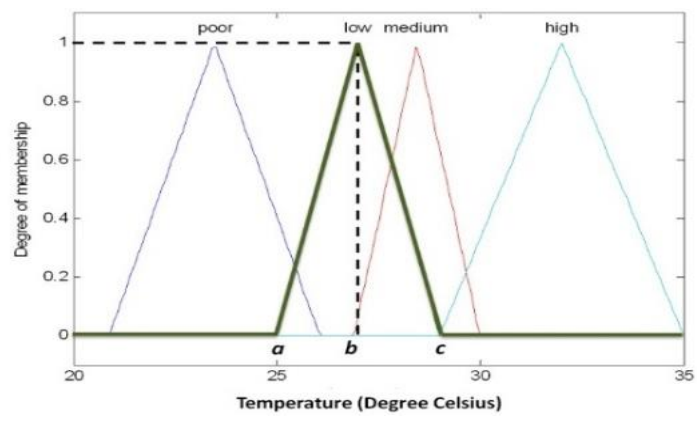

Figure 5. Triangular MF

Let $\tilde{A}=(a, b, c)$ with $\mathrm{a}<\mathrm{b}<\mathrm{c}$, be a fuzzy set on $R=(-\infty, \infty)$. It is called a triangular fuzzy number, if its MF is

$$
\mu_{\tilde{A}}(x)=\left\{\begin{array}{l}
\frac{x-a}{b-a}, \text { if } a \leq x \leq b \\
\frac{c-x}{c-b}, \text { if } b \leq x \leq c \\
0, \quad \text { otherwise }
\end{array}\right.
$$

The MFs have been defined and built by using weather parameters and the fuzzy output is rainfall rate. All membership function types were selected based on statistical study of training data, synoptic and climitological study of the area, however most of the decision rely on knowledge and statistical analysis. The MFs linguistic variables for the output were adopted from the Mamdani model. In order to make the inference rules reliable, the rainfall rate has been classified into five linguistic labels; Poor, Low, Medium, High and Very High. A composition method of fuzzy relations for output has been identified using the fuzzy c-means (FCM) clustering method. In FCM, each data belongs to a cluster as specified by a membership group [26], [27]. FCM algorithm uses the reciprocal of distances to decide the cluster centres. FCM use weight that minimize the total weighted mean-square error. The FCM permits each feature vector to belong to every cluster with a fuzzy value. The algorithm assigns a feature vector to a cluster according to the maximum weight of the feature vector against all clusters [26]. Table and Table 2 show the MF input and output. Figure is a fuzzy representation based on the input output MFs for temperature. 
Table 2. Membership Function Interval Values for Fuzzy Output Parameter

\begin{tabular}{cccc}
\hline Fuzzy Variable & \multicolumn{3}{c}{ Rainfall Rate } \\
\hline Poor & 0.01 & - & 3.09 \\
Low & 2.94 & - & 10.56 \\
Medium & 10.05 & - & 24.11 \\
High & 22.93 & - & 46.20 \\
Very High & 43.95 & - & 63.18 \\
\hline
\end{tabular}

Table 2. Membership Function Interval Values for Fuzzy Input Parameters

\begin{tabular}{cccccc}
\hline & Humidity & Temperature & Pressure & Wind Speed & Dew Point \\
\hline Poor & $70.1-82.6$ & $22.4-25.3$ & $1001-1010$ & $0.07-0.49$ & $20.9-24.0$ \\
Low & $78.6-90.1$ & $24.0-27.0$ & $1005-1012$ & $0.47-1.23$ & $22.8-25.0$ \\
Medium & $86.3-96.4$ & $25.7-29.4$ & $1007-1014$ & $1.17-2.14$ & $23.8-26.3$ \\
High & $91.7-100.6$ & $27.9-30.8$ & $1009-1016$ & $2.04-4.41$ & $25.0-26.8$ \\
\hline
\end{tabular}
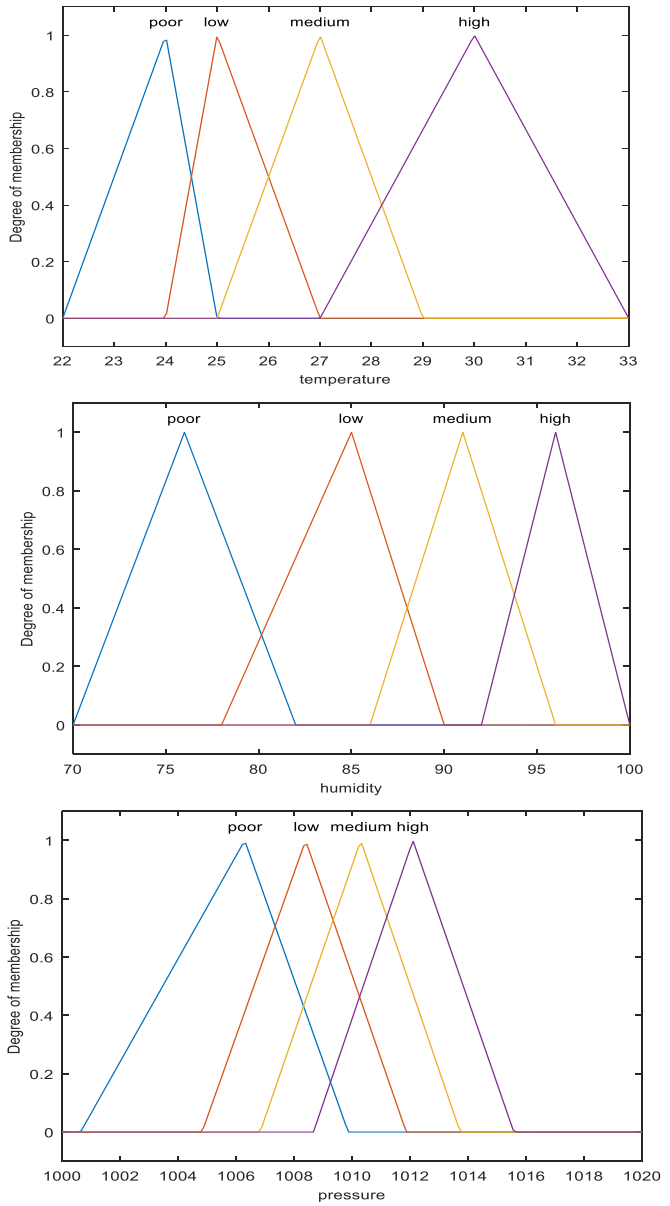
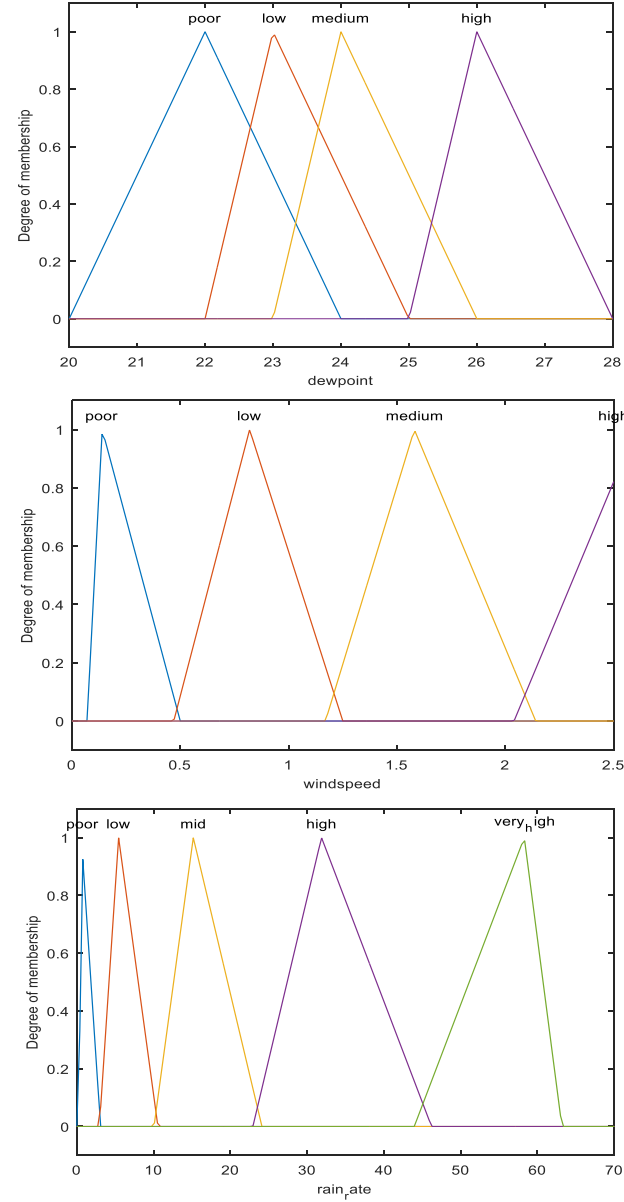

Figure 6. Input and output variables in Fuzzy representation

\subsubsection{IF THEN Rules Development and Fuzzy Set Operations}

The most intuitive approach in developing the rules from the fuzzy set is to list all possible combination of the input parameter's MF. The problems of listing all possible combination are that it makes the system too specific, waste system memory and increase chances of complete failure. Some rules may never be used. In order to reduce these problems, a proper classification technique to identify classes and their attribute is necessary. Decision learning tree technique is used to achieve this objective. The decision tree was proposed by Quinlan[28]. It is a projecting model that maps interpretations of an input to the target output. The induction of the rules to determine appropriate rainfall rate is an interactive process. In each process a new node (environmental parameter MF) is added. A node generates a number of sub-nodes until 
all the branches of the tree belong to the same class (rainfall rate). Figure shows the illustration of the decision tree process beginning with the root (Humidity is High).

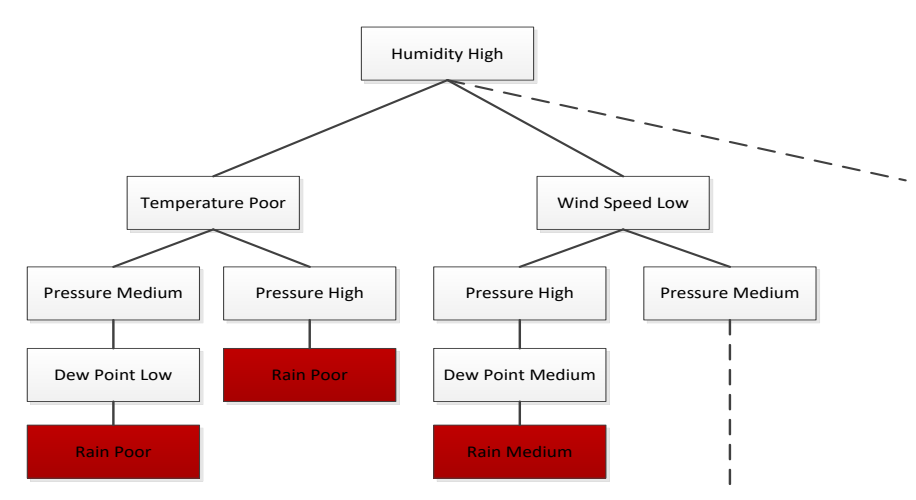

Figure 7. Fuzzy decision tree

\subsubsection{Inferences}

According to the empirical analysis of the association between the fuzzy input and fuzzy output mapping and the aggregation process of the output, 37 inference rules are generated in. Some of the inference rules as shown in Table 4.

Table 3. Some of the Inference Rules

\begin{tabular}{ccccccc}
\hline & Humidity & Temperature & Pressure & Wind Speed & Dew Point & Rain Rate Prediction \\
\hline Rule 1 & HIGH & POOR & MEDIUM & POOR & LOW & POOR \\
Rule 7 & HIGH & POOR & MEDIUM & POOR & MEDIUM & POOR \\
Rule 11 & HIGH & LOW & MEDIUM & LOW & MEDIUM & POOR \\
Rule 18 & MEDIUM & LOW & MEDIUM & LOW & HIGH & POOR \\
Rule 19 & HIGH & POOR & HIGH & NONE & HIGH & LOW \\
Rule 29 & MEDIUM & POOR & HIGH & NONE & LOW & LOW \\
Rule 30 & MEDIUM & LOW & HIGH & NONE & LOW & LOW \\
Rule 31 & HIGH & POOR & HIGH & LOW & MEDIUM & MEDIUM \\
Rule 36 & MEDIUM & LOW & HIGH & MEDIUM & MEDIUM & HIGH \\
Rule 37 & MEDIUM & NONE & MEDIUM & MEDIUM & MEDIUM & VERY HIGH \\
\hline
\end{tabular}

\subsubsection{Defuzzification}

Fuzziness benefit the system to evaluate the rules, but the final output of a fuzzy system has to be a crisp number [29]. It means that fuzzy numbers must be mapped to real values which can then be compared for magnitude. This assignment of a real value to a fuzzy number is called defuzzification. The input for the defuzzification process is the aggregated output from the fuzzy set meanwhile the output from the fuzzy system is the rainfall rate. Centroid technique is used in defuzzification process. The centre of gravity is the curve describing a given fuzzy quantity. Its computation requires integration of the membership functions. It finds the point where a vertical line would devide the aggregate set into two equal masses. Centroid defuzzification method finds a point representing the centre of the gravity of the fuzzy set of $x, A$, in the interval, $a$ to $b$. A reasonable estimate can be obtained by calculating it over a sample of points. Mathematically this centre of gravity can be expressed as:

$$
C O G=\frac{\int_{a}^{b} \mu_{A}(x) x d x}{\int_{a}^{b} \mu_{A}(x) d x}
$$

\section{PERFORMANCE INDICES}

Mean Absolute Error (MAE) and Root Mean Square Error (RMSE) were used to evaluate the performance of the outcome. 


$$
\begin{aligned}
& \text { MAE }=\frac{1}{n} \sum_{t=1}^{n}\left|y_{t}-\hat{y}_{t}\right| \\
& R M S E=\sqrt{\frac{1}{n} \sum_{t=1}^{n}\left(y_{t}-\hat{y}_{t}\right)^{2}}
\end{aligned}
$$

where $y_{t}$ is the real value, $\hat{y}_{t}$ is forecast value and $n$ is the number of observations.

The correlation coefficient between real and forecasted values of rainfall output were calculated. If each variable has $n$ observations, then the Pearson correlation coefficient of $R_{y_{t} \hat{y}_{t}}[30]$ is defined as:

$$
R_{y_{i}, \hat{y}_{t}}=\frac{\sum_{i=1}^{n}\left(y_{t i}-\bar{y}_{t}\right)\left(\hat{y}_{t i}-\overline{\hat{y}}_{t}\right)}{\sqrt{\sum_{i=1}^{n}\left(y_{t i}-\overline{y_{t}}\right)^{2}}-\sqrt{\sum_{i=1}^{n}\left(\hat{y}_{t i}-\overline{\hat{y}}_{t}\right)^{2}}}
$$

The $R$ value is an indication of the relationship between the forecasted and observed rainfall. If $R$ is closed to 1 , this indicates that there is a linear relationship and if $R$ is close to zero, then there is non-linear relationship.

\section{RESULTS AND DISCUSSION}

The goal of the study is to predict rainfall rate based on the relationship between environmental parameters. By following the procedures described in Section 3, the procedure started with preliminary implementation Comparison between the actual and predicted result is important to ascertain the accuracy of the proposed model. The initial IF-THEN rules were used to predict rain or no-rain. The initial rules are important for the rainfall rate prediction. The accuracy of the prediction was evaluated using test dataset. The prediction for rainfall rate yielded $72 \%$ accuracy and the magnitude of error is 0.226 and 0.457 for $M A E$ and $R M S E$ respectively.

Table shows the summarization of the results in this study. The prediction of the rainfall rate and actual data were carried out and compared.The results show that the FIS model is promising in predicting rainfall rate. It is very useful to identify the intensity of the rain using environmental parameters.

Table 5. Results of the Performance Index

\begin{tabular}{ccc}
\hline \multicolumn{2}{l}{ Fuzzy Inference Model } \\
\hline MAE & RMSE & $\mathrm{R}$ \\
0.226 & 0.457 & 0.285 \\
\hline
\end{tabular}

\section{CONCLUSION}

This paper introduced the intelligent model for rainfall event and its intensity prediction. The prediction model is rule out from the development of the FIS. Even though the finding so far not in a favour of reaching the appropriate accuracy for the rainfall estimation for a huge dataset, but using such techniques it is necessary to fuse the capabilities of expert opinion, theoretical studies and the accuracy of the computer systems by producing a reliable associative rules. In this model, the appropriate membership functions for all parameters and the process of developing efficient rules make the methodology easy to be driven and implemented. The models presented can be improved further by increasing the set of input parameters, adjusting the set of rules, hybriding with other computational methods and seasonal data. The results of this work suggest an open area for other environmental weather prediction such as flood, drought and environmental parameters.

\section{ACKNOWLEDGEMENTS}

This work is sponsored by Universiti Tun Hussein Onn Malaysia under TIER1 FASA 1/2007, UTHM research grant and Gates IT Sdn. Bhd 


\section{REFERENCES}

[1] Q. Song and B. S. Chissom, "Forecasting enrollments with fuzzy time series_part I," Fuzzy sets and systems, vol. 54, no. 1, pp. 1-9, 1993.

[2] A. I. T. T.O.Olatayo, "Statistical Modelling and Prediction of Rainfall Time Series Data," Global Journal of Computer Science and Technology, vol. 14, no. 1, 2014.

[3] M. S. K. Awan and M. M. Awais, "Predicting weather events using fuzzy rule based system," Applied Soft Computing, vol. 11, no. 1, pp. 56-63, Jan. 2011.

[4] S. Handoyo and Marji, "The fuzzy inference system with least square optimization for time series forecasting," Indonesian Journal of Electrical Engineering and Computer Science, vol. 11, no. 3, pp. 1015-1026, 2018.

[5] A. Agboola and A. Gabriel, "Development of a Fuzzy Logic Based Rainfall Prediction Model," International Journal of Engineering and Technology, vol. 3, no. 4, pp. 427-435, 2013.

[6] S. a. Asklany, K. Elhelow, I. K. Youssef, and M. Abd El-wahab, "Rainfall events prediction using rule-based fuzzy inference system," Atmospheric Research, vol. 101, no. 1-2, pp. 228-236, Jul. 2011.

[7] M. Hasan, X. F. Shi, T. Tsegaye, and N. U. Ahmed, "Rainfall Prediction Model Improvement by Fuzzy Set Theory," vol. 2013, no. January, pp. 1-11, 2013.

[8] N. Bartoletti, F. Casagli, S. Marsili-libelli, A. Nardi, and L. Palandri, "Environmental Modelling \& Software Datadriven rainfall / runoff modelling based on a neuro-fuzzy inference system," Environmental Modelling and Software, vol. 106, pp. 35-47, 2018.

[9] K. S. Polytechnic and M. Sciences, "Modeling Rainfall Prediction using Fuzzy Logic," vol. 1, no. 4, pp. 929-936, 2013.

[10] T. Thongwan, A. Kangrang, and S. Homwuttiwong, “An Estimation of Rainfall using Fuzzy Set-Genetic Algorithms Model," vol. 4, no. 1, pp. 77-81, 2011.

[11] K. Pravendra and K. Pradip, "Rainfall-Runoff Modeling Using Fuzzy Technique for a Small Watershed In Maharashtra, India," vol. 4, no. 3, pp. 388-394, 2013.

[12] J. Kajornrit, K. W. Wong, and C. C. Fung, "Rainfall Prediction in the Northeast Region of Thailand using Modular Fuzzy Inference System," pp. 10-15, 2012.

[13] D. Solomatine, L. See, and R. Abrahart, "Data-driven modelling: concepts, approaches and experiences, " Practical Hydroinformatics, pp. 17-31, 2008.

[14] P. Kr, "Simultaneous Prediction of Wind Speed and Direction by Evolutionary Fuzzy Rule Forest, "International Conference on Computational Science, ICCS 2017, 12-14 June 2017, pp. 1-10, 2017.

[15] G. K. Rahul and M. Khurana, "A Comparative Study Review of Soft Computing Approach in Weather Forecasting," International Journal of Soft Computing and Engineering (IJSCE), vol. 2, no. 5, pp. 295-299, 2012.

[16] L. Zadeh, "Fuzzy sets," Information and control, vol. 8, no. 3, pp. 338-353, 1965.

[17] H. M. Noor, D. Ndzi, G. Yang, and N. Z. Mohd Safar, "Rainfall-based river flow prediction using NARX in Malaysia," 2017, no. March, pp. 10-12.

[18] W. R. Ismail, K. R. Abu-Bakar, Z. A. Rahaman, N. Kamaruzzaman, A. B. Ali, and M. Mansor, "Storage of sediment and nutrients in littoral zones of a shallow tropical reservoir: a case of Timah Tasoh Reservoir, Perlis, Malaysia," IAHS PUBLICATION, pp. 263-270, 2002.

[19] N. Z. Mohd-Safar, D. Ndzi, D. Sanders, H. M. Noor, and L. M. Kamarudin, "Integration of Fuzzy C-Means and Artificial Neural Network for Short-Term Localized Rainfall Forecasting in Tropical Climate," in Intelligent Systems and Applications, 2018, pp. 325-348.

[20] N. Z. Mohd-Safar, D. Ndzi, I. Kagalidis, Y. Yang, and A. Zakaria, "Short-Term Localized Weather Forecasting by Using Different Artificial Neural Network Algorithm in Tropical Climate," in Proceedings of SAI Intelligent Systems Conference (IntelliSys) 2016: Volume 2, 2018, pp. 463-476.

[21] MATLAB, MATLAB. Natick, Massachusetts: The MathWorks Inc., 2012.

[22] E. H. Mamdani, "Advances in the linguistic synthesis of fuzzy controllers," International Journal of Man-Machine Studies, vol. 8, no. 6, pp. 669-678, 1976.

[23] S. Medasani and J. Kim, "An overview of membership function generation techniques for pattern recognition," vol. 19, pp. 391-417, 1998.

[24] A. Bárdossy, "Fuzzy Sets in Rainfall/Runoff Modeling," in Encyclopedia of Hydrological Sciences, American Cancer Society, 2006.

[25] E. Ghasemi, M. Ataei, and H. Hashemolhosseini, "Development of a fuzzy model for predicting ground vibration caused by rock blasting in surface mining," Journal of Vibration and Control, vol. 19, no. 5, pp. 755-770, 2013.

[26] J. C. Bezdek, "FCM:The Fuzzy C-Means Clustering Algorithm," Computers \& Geosciences, vol. 10, no. 2, pp. 191-203, 1984.

[27] M. Setnes, "Supervised Fuzzy Clustering for Rule Extraction," vol. 8, no. 4, pp. 416-424, 2000.

[28] J. R. Quinlan, "Induction of decision trees," Machine learning, vol. 1, no. 1, pp. 81-106, 1986.

[29] N. R. Sari, W. F. Mahmudy, A. P. Wibawa, E. Sonalitha, and N. R. Sari, "Enabling External Factors for Inflation Rate Forecasting using Fuzzy Neural System," Indonesian Journal of Electrical Engineering and Computer Science, vol. 7, no. 5, pp. 2746-2756, 2017.

[30] J. Hauke and T. Kossowski, "Comparison of Values of Pearson's and Spearman's Correlation Coefficients on the Same Sets of Data,” Quaestiones Geographicae, vol. 30, no. 2, pp. 87-93, 2011. 